\title{
Institutions for Behavioural Dynamic Logic with Binders
}

\author{
Rolf Hennicker ${ }^{1}$ and Alexandre Madeira ${ }^{2,3 \star}$ \\ 1 Ludwig-Maximilians-Universität München, Germany \\ 2 HASLab INESC TEC - Univ. Minho, Portugal \\ 3 CIDMA - Univ. Aveiro, Portugal
}

\begin{abstract}
Dynamic logic with binders $\mathcal{D}^{\downarrow}$ has been introduced as an institution for the development of reactive systems based on model class semantics. The satisfaction relation of this logic was, however, not abstract enough to enjoy the modal invariance property (bisimilar models should satisfy the same sentences). We recently overcame this problem by proposing an observational satisfaction relation where the equality on states is interpreted by bisimilarity of states. This entailed, however, a price to pay - the satisfaction condition required for institutions was lost. This paper works on this limitation by establishing a behavioural semantics for $\mathcal{D}^{\downarrow}$ parametric to behavioural structures - families of equivalence relations on the states of each model. Such structures are taken in consideration in the signature category and, in particular, for the definition of signature morphisms. We show that with these changes we get again an institution with a behavioural model class semantics. The framework is instantiated with specific behavioural structures, resulting in the novel Institution of Crucial Actions.
\end{abstract}

\section{Introduction}

This paper deals with logical formalisms for the specification and development of reactive systems. Dynamic logic with binders, called $\mathcal{D}^{\downarrow}$-logic, has been introduced in [13] as an institution in the sense of [6] which allows expressing properties of reactive systems, from abstract safety and liveness requirements down to concrete specifications of the (recursive) structure of executable processes. It is therefore well suited for program development by stepwise refinement. $\mathcal{D}^{\downarrow}$-logic combines modalities indexed by regular expressions of actions, as in Dynamic Logic [10], and state variables with binders, as in Hybrid Logic [4]. These motivations are reflected in its semantics. Differently from what is usual in modal logics, whose semantics is given by Kripke structures and satisfaction of formulas is evaluated globally, $\mathcal{D}^{\downarrow}$ models are reachable, labelled transition systems with initial states where satisfaction is evaluated. This reflects our focus on computations, i.e. on effective processes.

\footnotetext{
* This work is financed by the ERDF European Regional Development Fund through the Operational Programme for Competitiveness and Internationalisation - COMPETE 2020 Programme and by National Funds through the Portuguese funding agency, FCT - Fundação para a Ciência e a Tecnologia, within project POCI-01-0145-FEDER-016692 and by the individual grant SFRH/BPD/103004/2014.
} 


\subsection{Motivation}

The commitment of $\mathcal{D}^{\downarrow}$-logic concerning bisimulation equivalence is, however, not satisfactory: the model class semantics of specifications in $\mathcal{D}^{\downarrow}$ is not closed under bisimulation equivalence; there are $\mathcal{D}^{\downarrow}$-sentences that distinguish bisimulation equivalent models, i.e., $\mathcal{D} \downarrow$ does not enjoy the modal invariance property. As an example consider the two models $\mathcal{N}$ and $\mathcal{M}$ in Fig. 1 and the sentence $\downarrow x .\langle a\rangle x$. This sentence, evaluated in the initial state, expresses that after executing the action $a$ the initial state is reached again. Obviously this is true for $\mathcal{N}$ but not for $\mathcal{M}$ though $\mathcal{N}$ and $\mathcal{M}$ are bisimulation equivalent.

As a way out, we have proposed $\mathcal{D}_{\sim}^{\downarrow}$ logic [12] which relaxes the satisfaction relation such that equality of states is interpreted

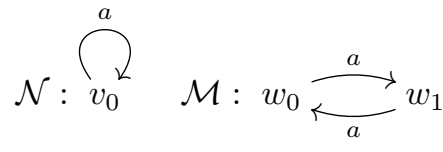

Fig. 1. Bisimilar models by bisimilarity of states. We call this observational equality and denote it by $\sim_{\mathcal{M}}$ for each model $\mathcal{M}$. Then the model $\mathcal{M}$ in Fig. 1 satisfies observationally the sentence $\downarrow x$. $\langle a\rangle x$, denoted by $\mathcal{M} \models \sim \downarrow x .\langle a\rangle x$, since the two states $w_{0}$ and $w_{1}$ are observationally equal. Indeed we have shown in [12] that in $\mathcal{D} \underset{\sim}{\downarrow}$ the modal invariance property holds. But, unfortunately, with relaxing the satisfaction relation we lost the institution property of $\mathcal{D}^{\downarrow}$ because $\mathcal{D} \underset{\sim}{\downarrow}$ does not satisfy the satisfaction condition of an institution. Intuitively the satisfaction condition expresses that truth is invariant under change of notation [6]. From the software engineer's perspective it expresses that satisfaction of properties, i.e. sentences, should be preserved when models are put in a larger context. Fig. 2 illustrates the problem with $\mathcal{D}_{\sim}^{\downarrow}$.

It shows two models $\mathcal{M}$ and $\mathcal{M}^{\prime}$. The signature of $\mathcal{M}$ is the singleton action set $A=$

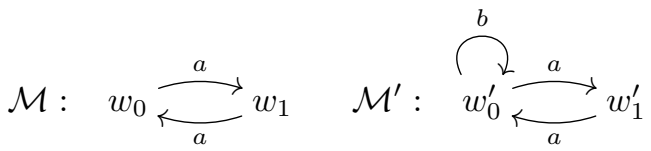

Fig. 2. Examples of $\{a\}$ and $\{a, b\}$-models $\{a\}$ and the signature of $\mathcal{M}^{\prime}$ is the larger action set $A^{\prime}=$ $\{a, b\}$. As a signature morphism we take the inclusion $\sigma: A \rightarrow A^{\prime}$ with $\sigma(a)=a$. Looking at $\mathcal{M}^{\prime}$ we see that $w_{0}^{\prime}$ and $w_{1}^{\prime}$ are not observationally equal, since in $w_{0}^{\prime}$ the action $b$ is enabled which is not the case in state $w_{1}^{\prime}$. Hence, $\mathcal{M}^{\prime} \not \models_{\sim} \downarrow x$. $\langle a\rangle x$. Restricting $\mathcal{M}^{\prime}$ to $A$ yields the $A$-model $\mathcal{M}$. As we have seen before $\mathcal{M} \mid \sim \downarrow x$. $\langle a\rangle x$. Hence, observational satisfaction is not preserved in larger contexts and therefore the satisfaction condition does not hold in $\mathcal{D}_{\sim}^{\downarrow}$. In this work, we are looking for possibilities to overcome this deficiency. 


\subsection{Overview of the Proposal}

On the way to solve the problem we found (again) the paper of Misiak [15] who has studied institutions with behavioural semantics in arbitrary logical systems with concrete model categories. Misiak's paper is an abstraction of more concrete institutions that have been studied in the framework of observational algebraic specification where a similar problem to ours was solved by adding restrictions to signature morphisms $[7,5]$ and putting more information into the signatures $[8$, 11,3]. To instantiate Misiak's approach by using labelled transition systems as models, we forget, for the moment, the observational equalities $\sim_{M}$ and consider instead, for each set of actions $A$, a family $\approx$ of equivalence relations $\approx_{\mathcal{M}}$, indexed by the models of $\mathcal{D}^{\downarrow}$. A signature is then a pair $(A, \approx)$ and the satisfaction of sentences is defined by interpreting equality of states in terms of $\approx_{\mathcal{M}}$. Misiak's trick is to consider a signature morphism as a mapping $\sigma: A \rightarrow A^{\prime}$ which is compatible with the equivalences of each signature. This means, more formally, that for each $A^{\prime}$-model $\mathcal{M}^{\prime}$ the restriction of $\approx_{\mathcal{M}^{\prime}}$ to the states of $\mathcal{M}$, denoted by $\left.\left(\approx_{\mathcal{M}^{\prime}}\right)\right|_{\sigma}$, is the same as the equivalence $\approx_{\left(\left.\mathcal{M}^{\prime}\right|_{\sigma}\right)}$ used in $\approx$ for the $A$-model $\left.\mathcal{M}^{\prime}\right|_{\sigma}$, which is the reduct of $\mathcal{M}^{\prime}$ along $\sigma$. This means that we have, for each $A^{\prime}$-model $\mathcal{M}^{\prime}$, the following crucial equation:

$$
\left.\left(\approx_{\mathcal{M}^{\prime}}\right)\right|_{\sigma}=\approx_{\left(\left.\mathcal{M}^{\prime}\right|_{\sigma}\right)}
$$

Thus the satisfaction condition is enforced by the notion of a signature morphism and we may ask how useful this additional information in signatures, given by the family of equivalences, can be for our problem. In particular, how the family of equivalences can be syntactically presented and how this can be related to the observational equalities $\sim_{\mathcal{M}}$ considered in $\mathcal{D} \underset{\sim}{\downarrow}$. To approach this, we first observe that Misiak has reduced the model classes for signatures $(A, \approx)$ to those models $\mathcal{M}$ for which the equivalence $\approx_{\mathcal{M}}$ is a congruence. ${ }^{4}$ In our framework of labelled transition systems this makes perfect sense, since the congruence property expresses that equivalence of states must be preserved when an action $a \in A$ is executed. In this way we obtain an institution. We also consider the "black-box" view of each $(A, \approx)$-model $\mathcal{M}$ obtained by its quotient structure $\mathcal{M} / \approx_{\mathcal{M}}$. We show that this construction can be extended to a full and faithfull functor mapping $(A, \approx)$-models to $A$-models in $\mathcal{D}^{\downarrow}$. This functor preserves and reflects satisfaction of sentences.

Next we are looking for a meaningful syntactic representation of signatures $(A, \approx)$. The idea comes from the observational algebraic specification frameworks $[8,11,3]$ where a distinguished subset of so-called observer operations has been selected for each signature. In our context of labelled transition systems we select, for each action set $A$, a distinguished subset $C \subseteq A$ of crucial actions and consider two states equivalent w.r.t. $C$ if they have the same behaviour under the execution of actions from $C$. This equivalence is called $(A, C)$-equality and denoted by $\sim_{\mathcal{M}}^{C}$ for each labelled transition system $\mathcal{M}$. Following the Misiak's

\footnotetext{
${ }^{4}$ Thus the information in signatures is used to constrain models as in [3]. In contrast, in Hiden Algebra [7] restrictions concern only signature morphisms but not models.
} 
approach we consider only those models $\mathcal{M}$ for which $\sim_{\mathcal{M}}^{C}$ is a congruence, i.e $\sim_{\mathcal{M}}^{C}$ is preserved by all actions in $A$. We show that in these models, called $(A, C)$ models, $(A, C)$-equality coincides with observational equality, i.e, $\sim_{\mathcal{M}}^{C}=\sim_{\mathcal{M}}$. The model class of $\mathcal{D}^{\downarrow}$ (and of $\mathcal{D} \underset{\sim}{\downarrow}$ ) is therefore restricted to those labelled transition systems for which the set of crucial actions is already sufficient to characterize the observational equality, i.e. bisimilarity of states. This has the side effect that for proving that two states are bisimilar it is sufficient to check transitions with actions from $\mathrm{C}$, a technique which has also been proposed in the selective $\mu$-calculus [2] to reduce verification complexity for modal formulae.

Having signatures as pairs $(A, C)$, it remains to define signature morphisms $\sigma:(A, C) \rightarrow\left(A^{\prime}, C^{\prime}\right)$ such that the equation (1) from above is valid for each $\left(A^{\prime}, C^{\prime}\right)$-model $\mathcal{M}^{\prime}$, which now means $\left.\left(\sim_{\mathcal{M}^{\prime}}\right)\right|_{\sigma}=\sim_{\left(\left.\mathcal{M}^{\prime}\right|_{\sigma}\right)}$ (with $\sim$ denoting observational equalities as before in $\left.\mathcal{D}_{\sim}^{\downarrow}\right)$. To achieve this, we require that no new crucial actions are introduced by $\sigma$, i.e., $\sigma[C]=C^{\prime}$. In this way, observational equalities are preserved under change of notation, in particular in larger contexts when the action set $A$ is enlarged to $A^{\prime}$. Hence the satisfaction condition holds and we get a concrete institution.

The paper is organized as follows. In Sect. 2, we recall the definitions of $\mathcal{D}^{\downarrow}$-logic and of the observational semantics defined in $\mathcal{D}_{\sim}^{\downarrow}$. Then, in Sect. 3, we apply Misiak's aproach to labelled transition systems yielding a behavioural institution with a family of congruence relations on models. We provide the black-box functor in Sect. 4, which maps the behavioural model category to the model category of $\mathcal{D}^{\downarrow}$-logic. In Sect. 5, we consider syntactic representations of signatures and signature morphisms leading to the crucial actions institution. We finish with concluding remarks in Sect. 6.

In order to fix notations, we recall here the institution definition from [6]: An institution $\mathcal{I}=\left(\operatorname{Sign}^{\mathcal{I}}, \operatorname{Sen}^{\mathcal{I}}, \operatorname{Mod}^{\mathcal{I}},\left(\mid={ }_{\Sigma}^{I}\right)_{\Sigma \in\left|\operatorname{Sign}^{\mathcal{I}}\right|}\right)$ consists of

- a category $\operatorname{Sign}^{\mathcal{I}}$ whose objects are signatures and arrows signature morphisms;

- a functor $\operatorname{Sen}^{\mathcal{I}}: \operatorname{Sign}^{\mathcal{I}} \rightarrow \mathbb{S e t}$ giving for each signature a set of sentences,

- a models functor $\operatorname{Mod}^{\mathcal{I}}:\left(\operatorname{Sign}^{\mathcal{I}}\right)^{o p} \rightarrow \mathbb{C}$ at, giving for each signature $\Sigma$ a category whose objects are $\Sigma$-models, and arrows are $\Sigma$-(model) homomorphisms; each arrow $\varphi: \Sigma \rightarrow \Sigma^{\prime} \in \operatorname{Sign}^{\mathcal{I}}$, (i.e., $\left.\varphi: \Sigma^{\prime} \rightarrow \Sigma \in\left(\operatorname{Sign}^{\mathcal{I}}\right)^{o p}\right)$ is mapped to a functor $\operatorname{Mod}^{\mathcal{I}}(\varphi): \operatorname{Mod}^{\mathcal{I}}\left(\Sigma^{\prime}\right) \rightarrow \operatorname{Mod}^{\mathcal{I}}(\Sigma)$ called reduct functor, whose effect is to cast a model of $\Sigma^{\prime}$ as a model of $\Sigma$;

- a satisfaction relation $\models_{\Sigma}^{I} \subseteq\left|\operatorname{Mod}^{\mathcal{I}}(\Sigma)\right| \times \operatorname{Sen}_{\mathcal{I}}^{\mathcal{I}}(\Sigma)$ for each $\Sigma \in\left|\operatorname{Sign}^{\mathcal{I}}\right|$, such that for each morphism $\varphi: \Sigma \rightarrow \Sigma^{\prime} \in \operatorname{Sign}^{\mathcal{I}}$, the satisfaction condition

$$
M^{\prime} \models{ }_{\Sigma^{\prime}}^{I} \operatorname{Sen}^{\mathcal{I}}(\varphi)(\rho) \text { iff } \operatorname{Mod}^{\mathcal{I}}(\varphi)\left(M^{\prime}\right) \models_{\Sigma}^{I} \rho
$$

holds for each $M^{\prime} \in\left|\operatorname{Mod}^{\mathcal{I}}\left(\Sigma^{\prime}\right)\right|$ and $\rho \in \operatorname{Sen}^{\mathcal{I}}(\Sigma)$.

\section{Dynamic Logics with Binders}

This section recalls the underlying definitions and facts of $\mathcal{D}^{\downarrow}$-logic introduced in [13] and its observational variant $\mathcal{D}_{\sim}^{\downarrow}$ introduced in [12]. While $\mathcal{D}^{\downarrow}$ is an institution, $\mathcal{D} \underset{\sim}{\downarrow}$ is not as explained before. 


\section{$2.1 \mathcal{D}^{\downarrow}$-Logic}

Signatures for $\mathcal{D}^{\downarrow}$ are finite sets $A$ of atomic actions, and a signature morphism $A \stackrel{\sigma}{\longrightarrow} A^{\prime}$ is a function $\sigma: A \rightarrow A^{\prime}$. Clearly, this entails a category denoted by $\operatorname{Sign}^{\mathcal{D}^{\downarrow}}$.

Definition 1 (Models and model morphisms). Let $A$ be a finite set of atomic actions. An A-model is triple $\left(W, w_{0}, R\right)$ where $W$ is a set of states, $w_{0} \in W$ is the initial state and $R=\left(R_{a} \subseteq W \times W\right)_{a \in A}$ is a family of transition relations such that, for each $w \in W$, there is a finite sequence of transitions $R_{a^{k}}\left(w^{k-1}, w^{k}\right), 1 \leq k \leq n$, with $w^{k} \in W, a^{k} \in A$, such that $w^{0}=w_{0}$ and $w^{n}=w$. Given two A-models $\mathcal{M}=\left(W, w_{0}, R\right)$ and $\mathcal{M}^{\prime}=\left(W^{\prime}, w_{0}^{\prime}, R^{\prime}\right)$, a model morphism $h: \mathcal{M} \rightarrow \mathcal{M}^{\prime}$ is a function $h: W \rightarrow W^{\prime}$ such that $h\left(w_{0}\right)=w_{0}^{\prime}$ and, for each $a \in A$, if $\left(w_{1}, w_{2}\right) \in R_{a}$ then $\left(h\left(w_{1}\right), h\left(w_{2}\right)\right) \in R_{a}^{\prime}$.

The class of $A$-models and $A$-model morphisms define a category denoted by $\operatorname{Mod}^{\mathcal{D}^{\downarrow}}(A)$. The identity morphisms $i d_{\mathcal{M}}$ are the identity functions.

Definition 2 (Model reduct). Let $A \stackrel{\sigma}{\longrightarrow} A^{\prime}$ be a signature morphism and $\mathcal{M}^{\prime}=\left(W^{\prime}, w_{0}^{\prime}, R^{\prime}\right)$ an $A^{\prime}$-model. The reduct of $\mathcal{M}^{\prime}$ is the $A$-model $\left.\mathcal{M}^{\prime}\right|_{\sigma}=$ $\left(\left.W^{\prime}\right|_{\sigma},\left.R^{\prime}\right|_{\sigma},\left.w_{0}^{\prime}\right|_{\sigma}\right)$ where $\left(\left.w_{0}^{\prime}\right|_{\sigma}\right)=w_{0}^{\prime}$ and $\left.W^{\prime}\right|_{\sigma}$ is the largest set with $\left.w_{0}^{\prime} \in W^{\prime}\right|_{\sigma}$. For each $\left.v \in W^{\prime}\right|_{\sigma}$, either $v=w_{0}^{\prime}$ or there is a $\left.w \in W^{\prime}\right|_{\sigma}$ such that $(w, v) \in R_{\sigma(a)}^{\prime}$, for some $a \in A$. For each $a \in A, R_{a}=R_{\sigma(a)}^{\prime} \cap(W \times W)$.

The reduct $\left.\right|_{\sigma}$ induces, for each signature morphism $\sigma: A \rightarrow A^{\prime}$, a functor $\operatorname{Mod}^{\mathcal{D}^{\downarrow}}(\sigma): \operatorname{Mod}^{\mathcal{D}^{\downarrow}}\left(A^{\prime}\right) \rightarrow \operatorname{Mod}^{\mathcal{D}^{\downarrow}}(A)$. This functor, named reduct functor, maps models as $\operatorname{Mod}^{\mathcal{D}^{\downarrow}}\left(\mathcal{M}^{\prime}\right)=\left.\mathcal{M}^{\prime}\right|_{\sigma}$ and $A^{\prime}$-model morphisms $h: \mathcal{M}^{\prime} \rightarrow \mathcal{N}^{\prime}$ to $A$ model morphisms $\left.h\right|_{\sigma}:\left.\left.\mathcal{M}^{\prime}\right|_{\sigma} \rightarrow \mathcal{N}^{\prime}\right|_{\sigma}$, where $\left.h\right|_{\sigma}$ is the restriction of $h$ to the scope of $\left.\mathcal{M}^{\prime}\right|_{\sigma}$ and $\left.\mathcal{N}^{\prime}\right|_{\sigma}$. Finally, we consider the contravariant models functor $\operatorname{Mod}^{\mathcal{D}^{\downarrow}}:\left(\operatorname{Sign}^{\mathcal{D}^{\downarrow}}\right)^{o p} \rightarrow \mathbb{C} a t$ that maps each signature to its model category and each signature morphism to the respective reduct functor.

Definition 3 (Formulas and sentences). The set of $A$-formulas $\operatorname{Fm}^{\mathcal{D}^{\downarrow}}(A)$ is given by

$$
\varphi::=\mathbf{t t}|\mathbf{f f}| x|\downarrow x . \varphi| @_{x} \varphi|\langle\alpha\rangle \varphi|[\alpha] \varphi|\neg \varphi| \varphi \wedge \varphi \mid \varphi \vee \varphi
$$

where $x \in X$, for $X$ an infinite set of variables, and actions are composed from atomic actions $a \in A$ by sequential composition choice and iteration:

$$
\alpha::=a|\alpha ; \alpha| \alpha+\alpha \mid \alpha^{*}
$$

An A-formula $\varphi$ is called an A-sentence if $\varphi$ contains no free variables. Free variables are defined as usual with $\downarrow$ being the unique operator binding variables. The set of $A$-sentences is denoted by $\operatorname{Sen}^{\mathcal{D}^{\downarrow}}(A)$. 
The binder operator $\downarrow x . \varphi$ assigns to the variable $x$ the current state of evaluation and evaluates $\varphi$. The operator $@_{x} \varphi$ evaluates $\varphi$ in the state assigned to $x$.

Each signature morphism $\sigma: A \rightarrow A^{\prime}$ can be extended to a formula translation function $\hat{\sigma}: \operatorname{Fm}^{\mathcal{D} \downarrow}(A) \rightarrow \mathrm{Fm}^{\mathcal{D} \downarrow}\left(A^{\prime}\right)$, that keeps variables and connectives and replaces each action $a$ by $\sigma(a)$. If we restrict $\hat{\sigma}$ to sentences we get the translation function $\operatorname{Sen}^{\mathcal{D}^{\downarrow}}(\sigma): \operatorname{Sen}^{\mathcal{D}^{\downarrow}}(A) \rightarrow \operatorname{Sen}^{\mathcal{D}^{\downarrow}}\left(A^{\prime}\right)$ with $\operatorname{Sen}^{\mathcal{D}^{\downarrow}}(\sigma)(\varphi)=\hat{\sigma}(\varphi)$ for $\varphi \in \operatorname{Sen}^{\mathcal{D}^{\downarrow}}(A)$. Hence we have the sentence functor $\operatorname{Sen}^{\mathcal{D}^{\downarrow}}: \operatorname{Sign}^{\mathcal{D}^{\downarrow}} \rightarrow$ Set , that maps each signature to the set of its sentences, and each signature morphism to the corresponding translation of sentences.

To define the satisfaction relation formally we need to clarify how composed actions are interpreted in models. Let $\alpha \in \operatorname{Act}(A)$ and $\mathcal{M} \in \operatorname{Mod}^{\mathcal{D} \downarrow}(A)$. The interpretation of an action $\alpha$ in $\mathcal{M}$ extends the interpretation of atomic actions by $R_{\alpha ; \alpha^{\prime}}=R_{\alpha} \cdot R_{\alpha^{\prime}}, R_{\alpha+\alpha^{\prime}}=R_{\alpha} \cup R_{\alpha^{\prime}}$ and $R_{\alpha^{*}}=\left(R_{\alpha}\right)^{\star}$, with the operations $\circ, \cup$ and $\star$ standing for relational composition, union and reflexive-transitive closure. For a set $X$ of variables and an $A$-model $\mathcal{M}=\left(W, w_{0}, R\right)$, a valuation is a function $g: X \rightarrow W$. Given such a valuation $g$, a variable $x \in X$ and a state $w \in W, g[x \mapsto w]$ denotes the valuation with $g[x \mapsto w](x)=w$ and $g[x \mapsto w](y)=g(y)$ for any $y \in X, y \neq x$. Given an $A$-model $\mathcal{M}=\left(W, w_{0}, R\right)$, $w \in W$ and $g: X \rightarrow W$,

- $\mathcal{M}, g, w \models \mathbf{t t}$ is true; $\mathcal{M}, g, w \models \mathrm{ff}$ is false;

$-\mathcal{M}, g, w \models x$ iff $g(x)=w$;

$-\mathcal{M}, g, w \models \downarrow x . \varphi$ iff $\mathcal{M}, g[x \mapsto w], w \models \varphi ;$

$-\mathcal{M}, g, w \models @_{x} \varphi$ iff $\mathcal{M}, g, g(x) \models \varphi$;

- $\mathcal{M}, g, w \models\langle\alpha\rangle \varphi$ iff there is a $v \in W$ with $(w, v) \in R_{\alpha}$ and $\mathcal{M}, g, v=\varphi$;

$-\mathcal{M}, g, w \models[\alpha] \varphi$ iff for any $v \in W$ with $(w, v) \in R_{\alpha}$ it holds $\mathcal{M}, g, v \models \varphi$;

- $\mathcal{M}, g, w \models \neg \varphi$ iff it is false that $\mathcal{M}, g, w=\varphi$;

- $\mathcal{M}, g, w \models \varphi \wedge \varphi^{\prime}$ iff $\mathcal{M}, g, w \models \varphi$ and $\mathcal{M}, g, w \models \varphi^{\prime}$;

- $\mathcal{M}, g, w \models \varphi \vee \varphi^{\prime}$ iff $\mathcal{M}, g, w \models \varphi$ or $\mathcal{M}, g, w \models \varphi^{\prime}$.

We write $\mathcal{M}, w \models \varphi$ if, for any valuation $g: X \rightarrow W$, we have $\mathcal{M}, g, w \models \varphi$. If $\varphi$ is an $A$-sentence, then the valuation is irrelevant, i.e., $\mathcal{M}, g, w \models \varphi$ iff $\mathcal{M}, w \models \varphi$. $\mathcal{M}$ satisfies an $A$-sentence $\varphi$, written $\mathcal{M}=\varphi$, if $\mathcal{M}, w_{0}=\varphi$.

Finally, as shown in [13], the satisfaction condition holds and therefore these ingredients constitute an institution in the sense of Goguen and Burstall [6]:

Theorem 1 (Satisfaction condition). For any signature morphism $A \stackrel{\sigma}{\longrightarrow} A^{\prime} \in \operatorname{Sign}^{\mathcal{D}^{\downarrow}}$, model $\mathcal{M}^{\prime} \in \operatorname{Mod}^{\mathcal{D}^{\downarrow}}\left(A^{\prime}\right)$ and sentence $\varphi \in \operatorname{Sen}^{\mathcal{D}^{\downarrow}}(A)$, we have

$$
\operatorname{Mod}^{\mathcal{D}^{\downarrow}}(\sigma)\left(\mathcal{M}^{\prime}\right) \models \varphi \text { iff } \mathcal{M}^{\prime} \models \operatorname{Sen}^{\mathcal{D} \downarrow}(\sigma)(\varphi) .
$$

\section{$2.2 \mathcal{D} \underset{\sim}{\downarrow}-\operatorname{logic}$}

In the observational variant of $\mathcal{D}^{\downarrow}$, called $\mathcal{D}_{\sim}^{\downarrow}$ [12], the signature category and the sentences are the same as in $\mathcal{D}^{\downarrow}$. Models are also the same, but model morphisms 
and the satisfaction relation are different. Both make use of the observational equality of states, denoted by $\sim_{\mathcal{M}}$ for any $A$-model $\mathcal{M}$. For $\mathcal{M}=\left(W, w_{0}, R\right)$, observational equality $w \sim_{\mathcal{M}} v$ holds for two states $w, v \in W$ if there exists a bisimulation relation $B \subseteq W \times W$ such that $(w, v) \in B$.

Definition 4 (Observational morphisms). Let $\mathcal{M}=\left(W, w_{0}, R\right)$ and $\mathcal{M}^{\prime}=$ $\left(W^{\prime}, w_{0}^{\prime}, R^{\prime}\right)$ be two A-models. An observational morphism $h: \mathcal{M} \rightarrow \mathcal{M}^{\prime}$ is a relation $h \subseteq W \times W^{\prime}$ containing $\left(w_{0}, w_{0}^{\prime}\right)$ such that the following conditions are satisfied:

1. For any $a \in A, w, v \in W, w^{\prime} \in W^{\prime}$ such that $\left(w, w^{\prime}\right) \in h$ :

if $(w, v) \in R_{a}$, then there is a $v^{\prime} \in W^{\prime}$ such that $\left(w^{\prime}, v^{\prime}\right) \in R_{a}^{\prime}$ and $\left(v, v^{\prime}\right) \in h$.

2. For any $w, v \in W, w^{\prime}, v^{\prime} \in W^{\prime}$ such that $\left(w, w^{\prime}\right) \in h$ and $\left(v, v^{\prime}\right) \in h$ : if $w \sim \mathcal{M} v$, then $w^{\prime} \sim \mathcal{M}^{\prime} v^{\prime}$.

3. For any $w, v \in W, w^{\prime} \in W^{\prime}$ such that $\left(w, w^{\prime}\right) \in h$ : if $w \sim_{\mathcal{M}} v$, then $\left(v, w^{\prime}\right) \in h$.

4. For any $w \in W, w^{\prime}, v^{\prime} \in W^{\prime}$ such that $\left(w, w^{\prime}\right) \in h$ : if $w^{\prime} \sim \mathcal{M}^{\prime} v^{\prime}$, then $\left(w, v^{\prime}\right) \in h$.

A novelty of this model category is that isomorphism corresponds to bisimulation equivalence of models (see [12]). The observational satisfaction relation $\mathcal{M}, g, w=\sim \varphi$ is defined exactly as $\models$ with the exception of the satisfaction for variables which relaxes their interpretation up to observational equality, i.e., for any valuation $g$ and state $w$,

$$
\mathcal{M}, g, w \models \sim x \text { iff } g(x) \sim \mathcal{M} w
$$

These two adjustments on $\mathcal{D}^{\downarrow}$ ensure that $\mathcal{D}_{\sim}^{\downarrow}$ has the Hennessy-Milner property: Modal invariance holds w.r.t. $\models \sim$ and two image-finite models satisfying w.r.t. $=\sim$ the same sentences are bisimulation equivalent; see [12]. However, as illustrated in Sect. 1.1, the satisfaction condition does not hold in $\mathcal{D}_{\sim}^{\downarrow}$, i.e. $\mathcal{D}_{\sim}^{\downarrow}$ is not an institution.

\section{Behavioural Institution}

To get a behavioural institution we use the ideas of Misiak [15] who has studied institutions with behavioural semantics in arbitrary logical systems with concrete model categories. More specifically, our model categories will contain as objects $A$-models, i.e. transition systems with labels from $A$. The behavioural semantics introduced in this section is not committed to the observational equality but, following Misiak's idea, to an arbitrary family of equivalence relations, called behavioural structure. It should however be pointed out that, in contrast to Misiak's approach, we can define an explicit satisfaction relation here due to the specific model category of labeled transition systems.

Definition 5. $A$ behavioural structure for a set of actions $A$, i.e. $A \in \operatorname{Sign}^{\mathcal{D}^{\downarrow}}$, is a family $\approx=\left(\approx_{\mathcal{M}}\right)_{\mathcal{M} \in \operatorname{Mod}^{\mathcal{D} \downarrow}(A)}$ of equivalence relations $\approx_{\mathcal{M}} \subseteq W \times W$. 
Definition 6 (Behavioural signatures and their morphisms). $A$ behavioural signature is a pair $(A, \approx)$ where $A \in \operatorname{Sign}^{\mathcal{D}^{\downarrow}}$ is a set of actions and $\approx$ is a behavioural structure for $A$. Given two behavioural signatures $(A, \approx)$ and $\left(A^{\prime}, \approx^{\prime}\right)$, a behavioural signature morphism $(A, \approx) \stackrel{\sigma}{\longrightarrow}\left(A^{\prime}, \approx^{\prime}\right)$ is a function $\sigma: A \rightarrow A^{\prime}$ such that for any $\mathcal{M}^{\prime} \in \operatorname{Mod}^{\mathcal{D} \downarrow}\left(A^{\prime}\right)$, we have $\approx_{\left(\left.\mathcal{M}^{\prime}\right|_{\sigma}\right)}=\left.\left(\approx_{\mathcal{M}^{\prime}}^{\prime}\right)\right|_{\sigma}$.

Lemma 1. The behavioural signatures with respective morphisms define a category. This category will be denoted by $\operatorname{Sign}^{B}$.

For each behavioural signature $(A, \approx)$, sentences are given by $A$-sentences as before. The equivalences $\approx_{\mathcal{M}}$ used in a behavioural signature $(A, \approx)$ need not to be congruence relations for all $A$-models $\mathcal{M}$. Following Misiak's approach, in the new behavioural model category over a signature $(A, \approx)$ only those $A$-models $\mathcal{M}$ are admitted as $(A, \approx)$-models, for which $\approx_{\mathcal{M}}$ is a congruence relation.

Definition $7((A, \approx)$-Models and their morphisms). An A-model $\mathcal{M} \in$ $\operatorname{Mod}^{\mathcal{D}^{\downarrow}}(A)$ is an $(A, \approx)$-model if $\approx_{\mathcal{M}}$ is a congruence, in the following sense: for any $a \in A$ and $w, w^{\prime}, v \in W$, if $w \approx_{\mathcal{M}} v$ and $\left(w, w^{\prime}\right) \in R_{a}$, then there is a $v^{\prime} \in W$ such that $\left(v, v^{\prime}\right) \in R_{a}$ and $w^{\prime} \approx_{\mathcal{M}} v^{\prime}$. The morphisms between $(A, \approx)$-models are like observational model morphisms in Def. 4, but observational equalities $\sim_{\mathcal{M}}$ are replaced by the congruences $\approx_{\mathcal{M}}$ for each $(A, \approx)$-model $\mathcal{M}$.

Lemma 2 . The class of $(A, \approx)$-models with their respective morphisms define a category. This category will be denoted by $\operatorname{Mod}^{B}(A, \approx)$.

The next lemma shows that the reduct functor for models in $\operatorname{Mod}^{\mathcal{D}^{\downarrow}}(A)$ leads to a reduct functor for models in $\operatorname{Mod}^{B}(A, \approx)$. This is important to get an institution. It follows from the definition of behavioural signature morphisms.

Lemma 3. Let $\sigma:(A, \approx) \rightarrow\left(A^{\prime}, \approx^{\prime}\right) \in \operatorname{Sign}^{B}$ be a behavioural signature morphism and $\mathcal{M}^{\prime}$ an $\left(A^{\prime}, \approx^{\prime}\right)$-model. Then, the $A$-model $\left.\mathcal{M}^{\prime}\right|_{\sigma}$ is an $(A, \approx)$-model.

Proof. We have to prove that $\approx_{\left(\left.\mathcal{M}^{\prime}\right|_{\sigma}\right)}$ is a congruence in $\left.\mathcal{M}^{\prime}\right|_{\sigma}$. Let us suppose $w, w^{\prime},\left.v \in W^{\prime}\right|_{\sigma}$ such that $w \approx_{\left(\left.\mathcal{M}^{\prime}\right|_{\sigma}\right)} w^{\prime}$ and $(w, v) \in\left(\left.R^{\prime}\right|_{\sigma}\right)_{a}=R_{\sigma(a)}^{\prime}$. Since $\sigma$ is a behavioural signature morphism we have that $\approx_{\left(\left.\mathcal{M}^{\prime}\right|_{\sigma}\right)}=\left.\left(\approx_{\mathcal{M}^{\prime}}^{\prime}\right)\right|_{\sigma}$. Hence, we have

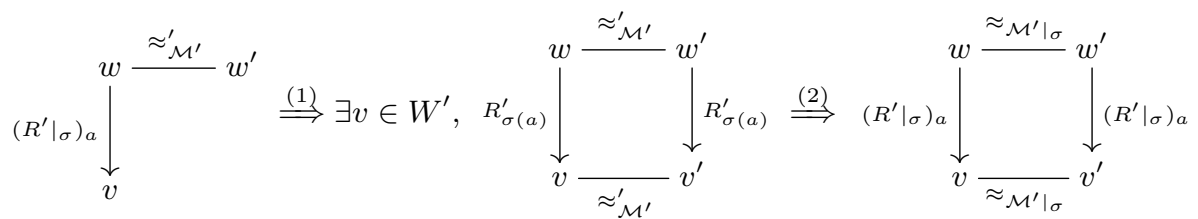

where (1) holds since $\approx_{\mathcal{M}^{\prime}}^{\prime}$ is a congruence in $\mathcal{M}^{\prime}$ and (2) holds, since $v^{\prime} \in W^{\prime}$ is accessible by $R_{\sigma(a)}$, thus $\left.v^{\prime} \in W^{\prime}\right|_{\sigma}$ and by the definition of $\left.R^{\prime}\right|_{\sigma}$.

As a consequence of the last lemma, we can use again the construction of reducts $\left.\right|_{\sigma}$ to define a models functor $\operatorname{Mod}^{B}:\left(\operatorname{Sign}^{B}\right)^{o p} \rightarrow \mathbb{C} a t$ similarly as done for $\mathcal{D}^{\downarrow}$ in Sect. 2.1. 
Definition 8 (Behavioural satisfaction $\models_{(A, \approx)}$ ). Let $\mathcal{M}$ be an $(A, \approx)$-model, $w \in W$ and $g: X \rightarrow W$ a valuation. The behavioural satisfaction of an $A$ formula $\varphi$ in state $w$ of $\mathcal{M}$ w.r.t. valuation $g$, denoted by $\mathcal{M}, g, w \models_{(A, \approx)} \varphi$, is defined analogously to the satisfaction relation $=$ in Sect. 2.1, with the exception of $\mathcal{M}, g, w \models_{(A, \approx)} x$ iff $g(x) \approx_{\mathcal{M}} w$. For A-sentences $\varphi$ valuations are irrelevant and we define $\mathcal{M} \models_{(A, \approx)} \varphi$, if $\mathcal{M}, w_{0} \models_{(A, \approx)} \varphi$.

The next theorem is the key to get the satisfaction relation. It relies on the definition of behavioural signature morphisms.

Theorem 2. Let $\sigma:(A, \approx) \rightarrow\left(A^{\prime}, \approx^{\prime}\right)$ be a signature morphism and $\mathcal{M}^{\prime}=$ $\left(W^{\prime}, w_{0}^{\prime}, R^{\prime}\right) \in \operatorname{Mod}^{B}\left(A^{\prime}, \approx^{\prime}\right)$. Then, for any $\left.w \in W^{\prime}\right|_{\sigma}\left(\subseteq W^{\prime}\right)$, for any valuation $g:\left.X \rightarrow W^{\prime}\right|_{\sigma}$, and for any A-formula $\varphi$,

$$
\operatorname{Mod}^{B}(\sigma)\left(\mathcal{M}^{\prime}\right), g, w=_{(A, \approx)} \varphi \text { iff } \mathcal{M}^{\prime}, g, w \models_{\left(A^{\prime}, \approx^{\prime}\right)} \hat{\sigma}(\varphi) .
$$

Proof. The proof is done by induction on the structure of formulas. We consider below atomic formulas $x, \downarrow x . \varphi$ and $\langle\alpha\rangle \varphi$. Actually, cases $\langle\alpha\rangle \varphi$ and $[\alpha] \varphi$ are proved similarly, and the remaining cases are trivial.

In the sequel we denote $\operatorname{Mod}^{B}(\sigma)\left(\mathcal{M}^{\prime}\right)$ by $\mathcal{M}$.

\section{Case $x$ :}

$$
\begin{aligned}
& \mathcal{M}, g, w \models_{(A, \approx)} x \\
& \Leftrightarrow \quad\left\{\models_{(A, \approx)} \text { def. }\right\} \\
& w \approx_{\mathcal{M}} g(x) \\
& \Leftrightarrow \quad\left\{\approx_{\mathcal{M}}=\left.\left(\approx_{\mathcal{M}^{\prime}}^{\prime}\right)\right|_{\sigma}\right\} \\
& \left.w\left(\approx_{\mathcal{M}^{\prime}}^{\prime}\right)\right|_{\sigma} g(x) \\
& \Leftrightarrow \quad\left\{w,\left.g(x) \in W^{\prime}\right|_{\sigma} \text { and by }\left.\right|_{\sigma} \text { def. }\right\} \quad \mathcal{M}^{\prime}, g, w \models_{\left(A^{\prime}, \approx^{\prime}\right)} \hat{\sigma}(x)
\end{aligned}
$$

Case $\downarrow x . \varphi$ :

$$
\begin{aligned}
& \mathcal{M}, g, w \models_{(A, \approx) \downarrow x . \varphi} \\
& \Leftrightarrow \quad\left\{\models_{(A, \approx)} \text { def. }\right\} \quad \Leftrightarrow \quad\left\{\models_{\left(A^{\prime}, \approx^{\prime}\right)} \text { def. }\right\} \\
& \mathcal{M}, g[x \mapsto w], w=_{(A, \approx)} \varphi \quad \mathcal{M}^{\prime}, g, w \models_{\left(A^{\prime}, \approx^{\prime}\right) \downarrow x . \hat{\sigma}(\varphi)} \\
& \Leftrightarrow \quad\{\text { I.H. }\} \quad \Leftrightarrow \quad\{\hat{\sigma} \text { def. }\} \\
& \mathcal{M}^{\prime}, g[x \mapsto w], w \models_{\left(A^{\prime}, \approx^{\prime}\right)} \hat{\sigma}(\varphi) \mid \mathcal{M}^{\prime}, g, w \models_{\left(A^{\prime}, \approx^{\prime}\right)} \hat{\sigma}(\downarrow x . \varphi)
\end{aligned}
$$

Case $\langle\alpha\rangle \varphi$ :

$$
\begin{aligned}
& \mathcal{M}, g, w \models_{(A, \approx)}\langle\alpha\rangle \varphi \\
& \Leftrightarrow \quad\left\{\models_{(A, \approx)} \text { def. }\right\} \\
& \mathcal{M}, g, v \models_{(A, \approx)} \varphi \text { for some }\left.v \in W^{\prime}\right|_{\sigma} \quad \Leftrightarrow \quad\left\{\models_{\left(A^{\prime}, \approx^{\prime}\right)} \text { def. }\right\} \\
& \text { such that }(w, v) \in\left(\left.R^{\prime}\right|_{\sigma}\right)_{\alpha} \\
& \Leftrightarrow \quad\{\operatorname{step}(\star)+\text { I.H. }\} \\
& \mathcal{M}^{\prime}, g, w \models_{\left(A^{\prime}, \approx^{\prime}\right)}\langle\sigma(\alpha)\rangle \hat{\sigma}(\varphi) \\
& \Leftrightarrow \quad\{\hat{\sigma} \text { def. }\} \\
& \mathcal{M}^{\prime}, g, w \models_{\left(A^{\prime}, \approx^{\prime}\right)} \hat{\sigma}(\langle\alpha\rangle \varphi)
\end{aligned}
$$


For the step ( $\star$ ) we just have to observe that for any action $\alpha \in \operatorname{Act}(A),\left.R^{\prime}\right|_{\alpha}=$ $R_{\bar{\sigma}(\alpha)}^{\prime} \cap\left(\left.W^{\prime}\right|_{\sigma}\right)^{2}$. This can be easily seen by induction on the structure of actions: The property holds by definition for basic actions $a \in A$. We consider below sequential composition of actions $\left(\alpha ; \alpha^{\prime}\right)$; the remainig cases follow a similar argument. So, we have $R_{\alpha ; \alpha^{\prime}}=R_{\alpha} \cdot R_{\alpha^{\prime}}={ }^{I . H .}\left(R_{\bar{\sigma}(\alpha)}^{\prime} \cap\left(\left.W^{\prime}\right|_{\sigma}\right)^{2}\right) \cdot\left(R_{\bar{\sigma}(\alpha)}^{\prime} \cap\left(\left.W^{\prime}\right|_{\sigma}\right)^{2}\right)$ Hence,

$$
\begin{aligned}
& (w, v) \in\left(R_{\bar{\sigma}(\alpha)}^{\prime} \cap\left(\left.W^{\prime}\right|_{\sigma}\right)^{2}\right) \cdot\left(R_{\bar{\sigma}(\alpha)}^{\prime} \cap\left(\left.W^{\prime}\right|_{\sigma}\right)^{2}\right) \\
\Leftrightarrow \quad & \quad\{\cdot \text { def. }\} \\
& (\exists z)\left((w, z) \in\left(R_{\bar{\sigma}(\alpha)}^{\prime} \cap\left(\left.W^{\prime}\right|_{\sigma}\right)^{2}\right) \wedge(z, v) \in\left(R_{\bar{\sigma}\left(\alpha^{\prime}\right)}^{\prime} \cap\left(\left.W^{\prime}\right|_{\sigma}\right)^{2}\right)\right) \\
\Rightarrow \quad & \quad\{\cdot \text { def. }+ \text { rewriting }\} \\
& (\exists z)\left(( w , z ) \in \left(R_{\bar{\sigma}(\alpha)}^{\prime} \wedge(z, v) \in R_{\left.\bar{\sigma}\left(\alpha^{\prime}\right)\right) \wedge}^{\prime}\right.\right. \\
& \left.(\exists z)\left((w, z) \in\left(\left.W^{\prime}\right|_{\sigma}\right)^{2} \wedge(z, v) \in\left(\left.W^{\prime}\right|_{\sigma}\right)^{2}\right)\right) \\
\Leftrightarrow \quad & \quad\{\cdot \text { def. }+ \text { rewriting }\} \\
& (w, v) \in\left(R_{\bar{\sigma}(\alpha)}^{\prime} \cdot R_{\bar{\sigma}\left(\alpha^{\prime}\right)}^{\prime}\right) \cap\left(\left(\left.W^{\prime}\right|_{\sigma}\right)^{2} \cdot\left(\left.W^{\prime}\right|_{\sigma}\right)^{2}\right) \\
\Rightarrow \quad & \quad\left\{\cap \text { monotonicity }\left(\text { since }\left(\left.W^{\prime}\right|_{\sigma}\right)^{2} \cdot\left(\left.W^{\prime}\right|_{\sigma}\right)^{2} \subseteq\left(\left.W^{\prime}\right|_{\sigma}\right)^{2}\right)+\bar{\sigma} \text { def. }\right\} \\
& (w, v) \in\left(R_{\bar{\sigma}\left(\alpha ; \alpha^{\prime}\right)}^{\prime}\right) \cap\left(\left.W^{\prime}\right|_{\sigma}\right)^{2}
\end{aligned}
$$

Therefore $R_{\alpha ; \alpha^{\prime}} \subseteq R_{\bar{\sigma}\left(\alpha ; \alpha^{\prime}\right)}^{\prime} \cap\left(\left.W^{\prime}\right|_{\sigma}\right)^{2}$. For the converse direction:

$$
\begin{aligned}
& R_{\bar{\sigma}\left(\alpha ; \alpha^{\prime}\right)}^{\prime} \cap\left(\left.W^{\prime}\right|_{\sigma}\right)^{2} \\
& =\{\bar{\sigma} \text { defn }+ \text { actions interpretation }\} \\
& \left(R_{\bar{\sigma}(\alpha)}^{\prime} \cdot R_{\bar{\sigma}\left(\alpha^{\prime}\right)}^{\prime}\right) \cap\left(\left.W^{\prime}\right|_{\sigma}\right)^{2} \\
& \subseteq \quad\{\cdot, \cap \text { distributivity }\} \quad R_{\alpha} \cdot R_{\alpha^{\prime}} \\
& \left(\left(R_{\bar{\sigma}(\alpha)}^{\prime} \cap\left(\left.W^{\prime}\right|_{\sigma}\right)^{2}\right) \cdot\left(R_{\bar{\sigma}\left(\alpha^{\prime}\right)}^{\prime} \cap\left(\left.W^{\prime}\right|_{\sigma}\right)^{2}\right)\right) \cap\left(\left.W^{\prime}\right|_{\sigma}\right)^{2}=\quad \text { \{ actions int. } \\
& =\{\text { I.H. }\} \\
& \mid \begin{array}{c}
\quad\left(R_{\alpha} \cdot R_{\alpha^{\prime}}\right) \cap\left(\left.W^{\prime}\right|_{\sigma}\right)^{2} \\
=\quad\left\{R_{\alpha}, R_{\alpha^{\prime}} \subseteq\left(\left.W^{\prime}\right|_{\sigma}\right)^{2}\right\} \\
R_{\alpha} \cdot R_{\alpha^{\prime}} \\
=\quad\{\text { actions int. }\} \\
R_{\alpha ; \alpha^{\prime}}
\end{array}
\end{aligned}
$$

Corollary 1 (Satisfaction condition). Let $\sigma:(A, \approx) \rightarrow\left(A^{\prime}, \approx^{\prime}\right)$ be a signature morphism and $\mathcal{M}^{\prime}=\left(W^{\prime}, w_{0}^{\prime}, R^{\prime}\right) \in \operatorname{Mod}^{B}\left(A^{\prime}, \approx^{\prime}\right)$. Then, for any $A$ sentence $\varphi$, we have $\operatorname{Mod}^{B}(\sigma)\left(\mathcal{M}^{\prime}\right) \models_{(A, \approx)} \varphi$ iff $\mathcal{M}^{\prime} \models_{\left(A^{\prime}, \approx^{\prime}\right)} \operatorname{Sen}^{B}(\sigma)(\varphi)$

Proof. This proof follows directly from Theorem 2: the satisfaction of sentences does not depend on the valuations (all the variables are bound and hence, their interpretation is determined by the model). Thus, for any state $\left.w \in W^{\prime}\right|_{\sigma}\left(\subseteq W^{\prime}\right)$ we have $\operatorname{Mod}^{B}(\sigma)\left(\mathcal{M}^{\prime}\right), w \models_{(A, \approx)} \varphi$ iff $\mathcal{M}^{\prime}, w \models_{\left(A^{\prime}, \approx^{\prime}\right)} \operatorname{Sen}^{B}(\sigma)(\varphi)$. Moreover, $\left.w_{0}^{\prime}\right|_{\sigma}=w_{0}^{\prime}$. Hence $\operatorname{Mod}^{B}(\sigma)\left(\mathcal{M}^{\prime}\right),\left.w_{0}^{\prime}\right|_{\sigma} \models_{(A, \approx)} \varphi$ iff $\mathcal{M}^{\prime}, w_{0}^{\prime} \models_{\left(A^{\prime}, \approx^{\prime}\right)}$ $\operatorname{Sen}^{B}(\sigma)(\varphi)$, i.e., $\operatorname{Mod}^{B}(\sigma)\left(\mathcal{M}^{\prime}\right) \models_{(A, \approx)} \varphi$ iff $\mathcal{M}^{\prime} \models_{\left(A^{\prime}, \approx^{\prime}\right)} \operatorname{Sen}^{B}(\sigma)(\varphi)$.

With the last corollary we have all ingredients to define the behavioural institution: 
Theorem 3. The tuple $B=\left(\operatorname{Sign}^{B}, \operatorname{Sen}^{\mathcal{D}^{\downarrow}}, \operatorname{Mod}^{B},(\models(A, \approx))_{\left((A, \approx) \in\left|\operatorname{Sign}^{B}\right|\right)}\right)$ is an institution.

\section{Black-Box Functor}

The black-box view of an $(A, \approx)$-model $\mathcal{M}$ is an $A$-model that represents the behaviour of $\mathcal{M}$ from the user's point of view. This model that collapses everything that is identified by $\approx_{\mathcal{M}}$, abstracting distinctions between states related by $\approx_{\mathcal{M}}$, is build via quotient construction. In this section we extend this construction to a full and faithful functor that maps each $(A, \approx)$-model into (an $A$-model representing) its black-box view. Finally we show that this functor preserves and reflects satisfaction of sentences.

Definition 9. Let $\mathcal{M}=\left(W, w_{0}, R\right)$ be an $(A, \approx)$-model. The quotient of $\mathcal{M}$, denoted by $\mathcal{M} / \approx_{\mathcal{M}}$, is the $A$-model $\left(W / \approx_{\mathcal{M}},\left[w_{0}\right]_{\approx_{\mathcal{M}}}, R / \approx_{\mathcal{M}}\right)$, where $W / \approx_{\mathcal{M}}=$ $\left\{[w]_{\approx_{\mathcal{M}}} \mid w \in W\right\}$ with $[w]_{\approx_{\mathcal{M}}}=\left\{w^{\prime} \in W \mid w \approx_{\mathcal{M}} w^{\prime}\right\}$ and

$\left(R / \approx_{\mathcal{M}}\right)_{a}=\left\{\left([w]_{\approx_{\mathcal{M}}},[v]_{\approx_{\mathcal{M}}}\right) \mid\right.$ there exist $w^{\prime} \in[w]_{\approx_{\mathcal{M}}}$ and $v^{\prime} \in[v]_{\approx_{\mathcal{M}}}$ s.t. $(w, v) \in$ $\left.R_{a}\right\}$.

Remark 1. For any $a \in A$ and $w, v \in W$, if $\left([w]_{\approx_{\mathcal{M}}},[v]_{\approx_{\mathcal{M}}}\right) \in\left(R / \approx_{\mathcal{M}}\right)_{a}$ then there exists $\hat{v} \in[v]$ such that $(w, \hat{v}) \in R_{a}$. This follows from the (zig) property of $\sim_{\mathcal{M}}$. This fact can be generalised to composed actions $\alpha \in \operatorname{Act}(A)$.

Definition 10. The Black Box map is defined as the pair of maps $\mathcal{B B}=\left(\mathcal{B B}_{\text {obj }}, \mathcal{B B}_{\text {hom }}\right)$ where $\mathcal{B B}_{\text {obj }}:\left|\operatorname{Mod}^{B}(A, \approx)\right| \rightarrow\left|\operatorname{Mod}^{\mathcal{D}^{\downarrow}}(A)\right|$ is a function defined for each $\mathcal{M} \in \operatorname{Mod}^{B}(A, \approx)$ by $\mathcal{B B}_{\text {obj }}(\mathcal{M})=\mathcal{M} / \approx_{\mathcal{M}}$; and $\mathcal{B B}_{\text {hom }}$ : $\operatorname{Hom}\left(\mathcal{M}, \mathcal{M}^{\prime}\right) \rightarrow \operatorname{Hom}\left(\mathcal{B B}(\mathcal{M}), \mathcal{B B}\left(\mathcal{M}^{\prime}\right)\right)$ a function mapping each morphism $h: \mathcal{M} \rightarrow \mathcal{M}^{\prime}$ to the relation $\mathcal{B B} h \subseteq W / \approx_{\mathcal{M}} \times W^{\prime} / \approx_{\mathcal{M}^{\prime}}$ defined by $\mathcal{B B} h=$ $\left\{\left([w]_{\approx_{\mathcal{M}}},\left[w^{\prime}\right]_{\approx_{\mathcal{M}^{\prime}}}\right) \mid\right.$ there are $v \in[w]_{\approx_{\mathcal{M}}}, v^{\prime} \in\left[w^{\prime}\right]_{\approx_{\mathcal{M}}}$ such that $\left.\left(v, v^{\prime}\right) \in h\right\}$. As usual, we omit in the sequel the subscripts in $\mathcal{B B}$.

Theorem 4. Black box is a functor $\mathcal{B B}: \operatorname{Mod}^{B}(A, \approx) \rightarrow \operatorname{Mod}^{\mathcal{D}^{\downarrow}}(A)$.

Proof. Let us firstly observe that $\mathcal{B B} h$ is a morphism in $\operatorname{Mod}^{\mathcal{D}^{\downarrow}}(A)$. According to Def. 1, we have to show that (i) it is a function and (ii) that it preserve transitions. In order to see (i), let us suppose $\left([w]_{\approx_{\mathcal{M}}},\left[w^{\prime}\right]_{\approx_{\mathcal{M}^{\prime}}}\right) \in \mathcal{B B} h$ and $\left([w]_{\approx_{\mathcal{M}}},\left[w^{\prime \prime}\right]_{\approx_{\mathcal{M}^{\prime}}}\right) \in \mathcal{B B} h$. By $\mathcal{B B} h$ definition we have that $\left(w, w^{\prime}\right) \in h$ and $\left(w, w^{\prime \prime}\right) \in h$. Since $h$ is an observational morphism, we have by 2 . of Def. 4 that $w^{\prime} \approx_{\mathcal{M}^{\prime}} w^{\prime \prime}$, and hence, $\left[w^{\prime}\right]_{\approx_{\mathcal{M}^{\prime}}}=\left[w^{\prime \prime}\right]_{\approx_{\mathcal{M}^{\prime}}} .^{5}$

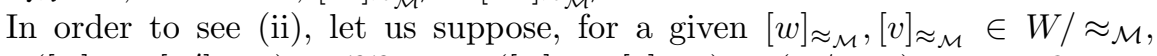
that $\left([w]_{\approx_{\mathcal{M}}},\left[w^{\prime}\right]_{\approx_{\mathcal{M}^{\prime}}}\right) \in \mathcal{B B h}$ and $\left([w]_{\approx_{\mathcal{M}}},[v]_{\approx_{\mathcal{M}}}\right) \in\left(R / \approx_{\mathcal{M}}\right)_{a}$. By definition of $\mathcal{B B h}$ and of $R / \approx_{\mathcal{M}}$, we have that $\left(w, w^{\prime}\right) \in h$ and $(w, v) \in R_{a}$. Moreover, since $h$ is a morphism we have by 1 . of Definition 4 that there is a $v^{\prime} \in W^{\prime}$ such

\footnotetext{
${ }^{5}$ For sake of uniformity, we still use along the section the relational notation to present this function, i.e. we use $\left(w, w^{\prime}\right) \in \mathcal{B B} h$ to represent $\mathcal{B B} h(w)=w^{\prime}$.
} 
that $\left(v, v^{\prime}\right) \in h$ and $\left(w^{\prime}, v^{\prime}\right) \in R_{a}^{\prime}$. Thus $\left(\left[w^{\prime}\right]_{\approx_{\mathcal{M}^{\prime}}},\left[v^{\prime}\right]_{\approx_{\mathcal{M}^{\prime}}}\right) \in\left(R^{\prime} / \approx_{\mathcal{M}^{\prime}}\right)_{a}$ and $\left([v]_{\approx_{\mathcal{M}}},\left[v^{\prime}\right]_{\approx_{\mathcal{M}^{\prime}}}\right) \in \mathcal{B B h}$.

Then, in order to be a functor we have also to see that, for any two morphisms $\mathcal{M} \stackrel{h}{\longrightarrow} \mathcal{M}^{\prime} \stackrel{h^{\prime}}{\longrightarrow} \mathcal{M}^{\prime \prime}, \mathcal{B B} h \cdot \mathcal{B B} h^{\prime}=\mathcal{B B} h \cdot h^{\prime}$. Then,

$$
\begin{aligned}
& \left([w]_{\approx_{\mathcal{M}}},\left[w^{\prime \prime}\right]_{\approx_{\mathcal{M}^{\prime \prime}}}\right) \in \mathcal{B B} h \cdot \mathcal{B B} h^{\prime} \\
& \Leftrightarrow \quad\{\text { relational composition }\} \\
& \exists\left[w^{\prime}\right]_{\approx_{\mathcal{M}}^{\prime}},\left([w]_{\approx_{\mathcal{M}}},\left[w^{\prime}\right]_{\approx_{\mathcal{M}^{\prime}}}\right) \in \mathcal{B B} h \\
& \text { and }\left(\left[w^{\prime}\right]_{\approx_{\mathcal{M}^{\prime}}},\left[w^{\prime \prime}\right]_{\approx_{\mathcal{M}^{\prime \prime}}}\right) \in \mathcal{B B} h^{\prime} \\
& \Leftrightarrow \quad\{\operatorname{Step}(\mathrm{a})\} \\
& \exists w^{\prime},\left(w, w^{\prime}\right) \in h \text { and }\left(w^{\prime}, w^{\prime \prime}\right) \in h^{\prime} \\
& \Leftrightarrow \quad\{\text { relational composition } \\
& \left(w, w^{\prime \prime}\right) \in h \cdot h^{\prime} \\
& \Leftrightarrow \quad\{\text { Step }(\mathrm{b})\} \\
& \left([w]_{\approx_{\mathcal{M}}},\left[w^{\prime \prime}\right]_{\approx_{\mathcal{M}^{\prime \prime}}}\right) \in \mathcal{B B} h \cdot h^{\prime}
\end{aligned}
$$

Step (a): implication $\Rightarrow$ : by definition of $\mathcal{B B}$ we have that there are $\bar{w} \in$ $[w]_{\approx_{\mathcal{M}}}, \bar{w}^{\prime} \in\left[w^{\prime}\right]_{\approx_{\mathcal{M}^{\prime}}}$ and $\bar{w}^{\prime \prime} \in\left[w^{\prime \prime}\right]_{\approx_{\mathcal{M}^{\prime \prime}}}$ such that $\left(\bar{w}, \bar{w}^{\prime}\right) \in h$ and $\left(\bar{w}^{\prime}, \bar{w}^{\prime \prime}\right) \in h^{\prime}$. But we have also that $\bar{w} \approx_{\mathcal{M}} w, \bar{w}^{\prime} \approx_{\mathcal{M}^{\prime}} w^{\prime}$ and $\bar{w}^{\prime \prime} \approx_{\mathcal{M}^{\prime \prime}} w^{\prime \prime}$. The implication follows by 3 . and 4 . of Def. 4 of the morphism $h$. Definition of $\mathcal{B B}$ entails the implication $\Leftarrow$. Justification of Step (b) is analogous. Moreover, $\mathcal{B B} 1_{\mathcal{M}}=$ $\left\{\left([w]_{\approx_{\mathcal{M}}},[v]_{\approx_{\mathcal{M}}}\right) \mid(w, v) \in 1_{\mathcal{M}}\right\}=\left\{\left([w]_{\approx_{\mathcal{M}}},[v]_{\left.\approx_{\mathcal{M}}\right)}\right) \mid w \approx_{\mathcal{M}} v\right\}=1_{\mathcal{B B}(\mathcal{M})}$.

Given a model $\mathcal{M} \in \operatorname{Mod}^{B}(A, \approx), \mathcal{B B}(\mathcal{M})$ is called black box view of $\mathcal{M}$.

Theorem 5. The functor $\mathcal{B B}$ is full.

Proof. Let us prove that $\mathcal{B B}$ is full, i.e. that for any morphism $k: \mathcal{B B}(\mathcal{M}) \rightarrow$ $\mathcal{B B}\left(\mathcal{M}^{\prime}\right)$ there is an observational morphism $h: \mathcal{M} \rightarrow \mathcal{M}^{\prime}$ such that $k=\mathcal{B B} h$. Let us consider the relation $h \subseteq W \times W^{\prime}=\left\{\left(v, v^{\prime}\right) \mid\left([w]_{\approx_{\mathcal{M}}},\left[w^{\prime}\right]_{\approx_{\mathcal{M}^{\prime}}}\right) \in k, v \in\right.$ $\left.[w]_{1_{\mathcal{M}}}, v^{\prime} \in\left[w^{\prime}\right]_{1_{\mathcal{M}^{\prime}}}\right\}$ It is enough to prove that $h$ is an observational morphism. Let us check the conditions of Def. 4: In order to see the condition 1.: by assuming $(v, r) \in R_{a}$ and $\left(v, v^{\prime}\right) \in h$, we have by definitions of $h$ and $R / \approx_{\mathcal{M}}$ that

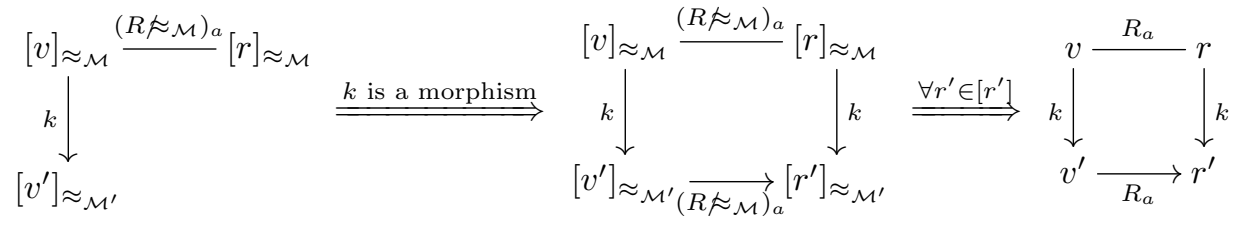

Conditions 2.,3. and 4. follow trivially, since $v \approx_{\mathcal{M}} r$ implies that $[v]_{\approx_{\mathcal{M}}}=[r]_{\approx_{\mathcal{M}}}$.

Theorem 6. The functor $\mathcal{B B}$ is faithful.

Proof. We have to show that, for any observational morphisms $h, h^{\prime}: \mathcal{M} \rightarrow \mathcal{M}^{\prime}$, $\mathcal{B B} h=\mathcal{B B} h^{\prime}$ implies $h=h^{\prime}$. In view of contradiction, let us suppose that $\mathcal{B B} h=\mathcal{B B} h^{\prime}$ and $h \neq h^{\prime}$. Then, there is a pair $\left(w, w^{\prime}\right)$ such that $\left(w, w^{\prime}\right) \in h$ and $\left(w, w^{\prime}\right) \notin h^{\prime}$ (or vice-versa). By $\mathcal{B B}$ definition we have $\left([w]_{\approx_{\mathcal{M}}},\left[w^{\prime}\right]_{\approx_{\mathcal{M}^{\prime}}}\right) \in$ $\mathcal{B B} h^{\prime}(=\mathcal{B B} h)$. Hence, there is an $r \in[w]_{\approx_{\mathcal{M}}}$ and $r^{\prime} \in\left[w^{\prime}\right]_{\approx_{\mathcal{M}^{\prime}}}$ such that $\left(r, r^{\prime}\right) \in$ $h^{\prime}$. Since $r \approx_{\mathcal{M}} w$ and $h^{\prime}$ is a morphism, we have by 3. of Def. 4 that $\left(w, r^{\prime}\right) \in h^{\prime}$. Moreover, since $r^{\prime} \approx_{\mathcal{M}^{\prime}} w^{\prime}$, we have by 4 . of Def. 4 that $\left(w, w^{\prime}\right) \in h^{\prime}$, what contradicts our initial assumption. Therefore $h=h^{\prime}$. 
Theorem 7. Let $\mathcal{M} \in \operatorname{Mod}^{B}(A, \approx)$ be a model. Then,

$$
\mathcal{M} \text { iso } \sim \mathcal{M}^{\prime} \text { iff } \mathcal{M} / \approx_{\mathcal{M}} \text { iso } \mathcal{M}^{\prime} / \approx_{\mathcal{M}^{\prime}} .
$$

Proof. Implication ' $\Rightarrow$ ' holds since $\mathcal{B B}$ is a functor. Implication ' $\Leftarrow$ ' is entailed because $\mathcal{B B}$ is a full and faithful functor

In the remainder of this section we show that the functor $\mathcal{B B}$ preserves and reflects satisfaction. This result is a simple generalisation of Theorem 5 in [12].

Theorem 8. For any model $\mathcal{M} \in \operatorname{Mod}^{B}(A, \approx)$ and for any $A$-sentence $\varphi$,

$$
\mathcal{M} \models_{(A, \approx)} \varphi \text { iff } \mathcal{M} / \approx_{\mathcal{M}}=\varphi
$$

Proof. For the proof we show, more generally, that for any $w \in W$, valuation $g: X \rightarrow W$ and $A$-formula $\varphi$,

$$
\mathcal{M}, g, w \models_{(A, \approx)} \varphi \text { iff } \mathcal{M} / \approx_{\mathcal{M}}, g / \approx_{\mathcal{M}},[w]_{\approx_{\mathcal{M}}} \models \varphi
$$

where $g / \approx_{\mathcal{M}}: X \rightarrow W$ is defined by $\left(g / \approx_{\mathcal{M}}\right)(x)=[g(x)]_{\approx_{\mathcal{M}}}$. The proof can be performed by induction over the structure of $A$-formulas. For the base formulas $\varphi=x$, we have:

$$
\begin{aligned}
& \mathcal{M}, g, w \models_{(A, \approx)} x \\
& \Leftrightarrow \quad\left\{\models_{(A, \approx)} \text { def. }\right\} \\
& \begin{array}{ll|l}
g(x) & \approx_{\mathcal{M}} w \\
\Leftrightarrow \quad\{\text { equivalence classes def. }\}
\end{array} \Leftrightarrow \quad \begin{aligned}
\left\{[g(x)]_{\approx_{\mathcal{M}}}=\left(g / \approx_{\mathcal{M}}\right)(x)\right. \\
\mathcal{M} / \approx_{\mathcal{M}}, g / \approx_{\mathcal{M}},[w]_{\mathcal{M}} \models x
\end{aligned}
\end{aligned}
$$

For the case $\varphi=\langle\alpha\rangle \phi$, we have:

$$
\begin{aligned}
& \mathcal{M}, g, w=_{(A, \approx)}\langle\alpha\rangle \phi \\
\Leftrightarrow & \quad\left\{=_{(A, \approx)} \text { def. }\right\} \\
& \text { there exists } v \in W \text { with }(w, v) \in R_{\alpha} \text { and } \mathcal{M}, g, v \mid=_{(A, \approx)} \phi \\
\Leftrightarrow \quad & \quad \text { step } \star\} \\
& \text { there exists }\left[v^{\prime}\right]_{\approx_{\mathcal{M}}} \in W / \approx_{\mathcal{M}} \text { with } \\
& \left([w]_{\approx_{\mathcal{M}}},\left[v^{\prime}\right]_{\approx_{\mathcal{M}}}\right) \in\left(R / \approx_{\mathcal{M}}\right)_{\alpha} \text { and } \mathcal{M} / \approx_{\mathcal{M}}, g / \approx_{\mathcal{M}},\left[v^{\prime}\right]_{\approx_{\mathcal{M}}} \models \phi \\
\Leftrightarrow \quad & \{\models \text { def. }\} \\
& \mathcal{M} / \approx_{\mathcal{M}}, g / \approx_{\mathcal{M}},[w]_{\approx_{\mathcal{M}}} \models\langle\alpha\rangle \phi
\end{aligned}
$$

Step $\star$ : The direction " $\Rightarrow$ " is trivial using $v^{\prime}=v$ and the Induction Hypothesis. For the direction " $\Leftarrow$ " assume $\left([w]_{\approx_{\mathcal{M}}},\left[v^{\prime}\right]_{\approx_{\mathcal{M}}}\right) \in\left(R / \approx_{\mathcal{M}}\right)_{\alpha}$ for some $v^{\prime}$. By Remark 1 we know that there exists $\hat{v} \in\left[v^{\prime}\right]_{\approx_{\mathcal{M}}}$ such that $(w, \hat{v}) \in R_{\alpha}$. From $\mathcal{M} / \approx_{\mathcal{M}}, g / \approx_{\mathcal{M}},\left[v^{\prime}\right]_{\approx_{\mathcal{M}}}=\phi$ it follows that $\mathcal{M} / \approx_{\mathcal{M}}, g / \approx_{\mathcal{M}},[\hat{v}]_{\approx_{\mathcal{M}}} \models \phi$ (since $\left.[\hat{v}]_{\approx_{\mathcal{M}}}=\left[v^{\prime}\right]_{\approx_{\mathcal{M}}}\right)$. By Ind. Hyp. we get $\mathcal{M}, g, \hat{v} \models_{(A, \approx)} \phi$. Since $(w, \hat{v}) \in R_{\alpha}$, we have $\mathcal{M}, g, w \models_{(A, \approx)}\langle\alpha\rangle \phi$.

The remaining cases are straightforward. 


\section{Institution of Crucial Actions}

This section introduces the "Logic of Crucial Actions". We show that this logic is a specific institution of observational dynamic logic with binders, inheriting the whole theory developed in the previous sections. The crucial idea to do this is to define signatures and signature morphisms syntactically and to relate them to behavioural signatures and behavioural signature morphisms as considered in Sect. 3. An important extra ingredient is that the restriction of $A$-models to those on which the given equivalences are congruences will yield, in the case of crucial actions signatures, exactly observational equalities. Thus, by applying the results of Sect. 3, we have recovered the satisfaction condition for the satisfaction relation $=\sim$ used in $\mathcal{D}_{\sim}^{\downarrow}$, since signatures with crucial actions have less models than in $\mathcal{D}_{\sim}^{\downarrow}$.

Definition 11 (Crucial actions signatures and morphisms). $A$ crucial actions signature is a pair $(A, C)$ where $A$ is a set of actions, and $C \subseteq A$ is a set of crucial actions. Given two crucial actions signatures $(A, C)$ and $\left(A^{\prime}, C^{\prime}\right)$, $a$ crucial actions signature morphism $\sigma:(A, C) \rightarrow\left(A^{\prime}, C^{\prime}\right)$ is a function $\sigma$ : $A \rightarrow A^{\prime}$ such that $\sigma[C]=C^{\prime}$.

Lemma 4. Crucial action signatures with their morphisms define a category. This category will be denoted by $\operatorname{Sign}^{C r}$.

Sentences of this logic are the same as in $\mathcal{D}_{\sim}^{\downarrow}$ and in $\mathcal{D}^{\downarrow}$. The sentences functor $\mathrm{Sen}^{C r}: \mathrm{Sign}^{C r} \rightarrow$ Set is defined as $\mathrm{Sen}^{\mathcal{D}^{\downarrow}}$ by forgetting the second component of the signatures. Now, we define a variant of bisimulation on $A$-models which takes into account only crucial actions in $C$. In the particular case where $C=A$ we get the usual notion of (strong) bisimulation.

Definition 12 (Crucial actions bisimulation). Let $(A, C)$ be a crucial actions signature and let $\mathcal{M}=\left(W, w_{0}, R\right)$ be an $A$-model. An $(A, C)$-bisimulation on $\mathcal{M}=\left(W, w_{0}, R\right)$ is a relation $B \subseteq W \times W$ such that $\left(w_{0}, w_{0}\right) \in B$ and

(zig) For any $c \in C, w, v, w^{\prime} \in W$ such that $\left(w, w^{\prime}\right) \in B$, if $(w, v) \in R_{c}$, then there is a $v^{\prime} \in W$ such that $\left(w^{\prime}, v^{\prime}\right) \in R_{c}$ and $\left(v, v^{\prime}\right) \in B$.

(zag) For any $c \in C, w, v, v^{\prime} \in W$ such that $\left(w, w^{\prime}\right) \in B$, if $\left(w^{\prime}, v^{\prime}\right) \in R_{c}^{\prime}$, then there is a $v \in W$ such that $(w, v) \in R_{c}$ and $\left(v, v^{\prime}\right) \in B$.

Definition $13((A, C)$-Equality). Let $(A, C)$ be a crucial actions signature. For any $A$-model $\mathcal{M}$ the $(A, C)$-equality on $\mathcal{M}$ is the relation $\sim_{\mathcal{M}}^{C} \subseteq W \times W$ such that, for any $w, w^{\prime}, w \sim_{\mathcal{M}}^{C} w^{\prime}$ iff there is an $(A, C)$-bisimulation $B$ in $\mathcal{M}$ such that $\left(w, w^{\prime}\right) \in B$.

Lemma 5. The family $\sim^{C}=\left(\sim_{\mathcal{M}}^{C}\right)_{\mathcal{M} \in \operatorname{Mod}^{\mathcal{D} \downarrow}(A)}$ is a behavioural structure.

Proof. For any $A$-model $\mathcal{M}$, the $(A, C)$-equality $\sim_{\mathcal{M}}^{C}$ is an equivalence relation. 
Given a crucial actions signature $(A, C)$, we consider, along the lines of Sect. 3, only those $A$-models $\mathcal{M}$ as admissible $(A, C)$-models, for which the $(A, C)$-equality $\sim_{\mathcal{M}}^{C}$ is a congruence.

Definition 14 (( $A, C)$-Models). $A$ model $\mathcal{M} \in \operatorname{Mod}^{\mathcal{D} \downarrow}(A)$ is an $(A, C)$-model if $\sim_{\mathcal{M}}^{C}$ is a congruence relation on $\mathcal{M}$.

Example 1. Let $A^{\prime}=\{a, b\}$ and $C^{\prime}=\{a\}$. The $A^{\prime}$-model $\mathcal{M}^{\prime}$ of Fig. 2 is not an $\left(A^{\prime}, C^{\prime}\right)$-model since we have $w_{0}^{\prime} \sim_{\mathcal{M}^{\prime}}^{C^{\prime}} w_{1}^{\prime}$ and $\left(w_{0}^{\prime}, w_{1}^{\prime}\right) \in R_{b}^{\prime}$ but action $b$ is not enabled in $w_{1}^{\prime}$. Now, consider the $A^{\prime}$-model $\mathcal{M}^{\prime \prime}$ in Fig. 3 . It is obviously an $(\{a, b\},\{a\})$-model.

The important point to link the current notions to the observational equality $\sim_{\mathcal{M}}$ considered in $\mathcal{D}_{\sim}^{\downarrow}$ is given by the next lemma.

Lemma 6. Let $\mathcal{M}$ be an A-model. $\mathcal{M}$ is an $(A, C)$-model if, and only if, the $(A, C)$-equality on $\mathcal{M}$ coincides with the observational equality $\sim_{\mathcal{M}}$, i.e. $\sim_{\mathcal{M}}^{C}=\sim_{\mathcal{M}}$.

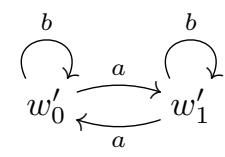

Fig. 3. $(\{a, b\},\{a\})$-model

Proof. The implication " $\Leftarrow$ " is easy since the observational equality $\sim_{\mathcal{M}}$ is trivially a congruence relation and so is $\sim_{\mathcal{M}}^{C}$ by assumption. For the implication " $\Rightarrow$ " we have to prove that $\sim_{\mathcal{M}}=\sim_{\mathcal{M}}^{C}$. The inclusion $\sim_{\mathcal{M}} \subseteq \sim_{\mathcal{M}}^{C}$ is obvious - we have the bisimulation properties assured for all actions of $A$ and hence also for all actions of $C$. For the converse inclusion, we observe that for any congruence $\equiv$ in $\mathcal{M}, w_{0} \equiv w_{0}$ (any congruence is an equivalence relation). Moreover, by definition, it satisfies ( $z i g$ ) and, because of its symmetry (any congruence is an equivalence relation), ( $z a g)$ also holds. Therefore any congruence $\equiv$ in $\mathcal{M}$ is a bisimulation in $\mathcal{M}$. Thus, since $\sim_{\mathcal{M}}^{C}$ is a congruence and by bisimulation equivalence definition, we have $\sim_{\mathcal{M}}^{C} \subseteq \sim_{\mathcal{M}}$.

Example 2. Consider again the $A^{\prime}$-models $\mathcal{M}^{\prime}$ and $\mathcal{M}^{\prime \prime}$ of the previous example. Actually, we have that the observational equality $\sim_{\mathcal{M}^{\prime}}$ is the identity of states in $\mathcal{M}^{\prime}$, while the crucial actions equality $\sim_{\mathcal{M}^{\prime}}^{C^{\prime}}$ identifies all states of $\mathcal{M}^{\prime}$. Due to the previous lemma, this shows again that $\mathcal{M}^{\prime}$ is not an $\left(A^{\prime}, C^{\prime}\right)$-model. Considering $\mathcal{M}^{\prime \prime}$, however, we have $\sim_{\mathcal{M}^{\prime \prime}}=\sim_{\mathcal{M}^{\prime \prime}}^{C^{\prime}}$ since the execution of action $b$ does not distinguish more elements than distinguished by $a$.

For each crucial actions signature $(A, C)$ we have the category of models $\operatorname{Mod}^{C r}(A, C)=\operatorname{Mod}^{B}\left(A, \sim^{C}\right)$. Next we show that crucial actions signature morphisms are behavioural signature morphisms in the sense of Def. 6 .

Lemma 7. Let $\sigma:(A, C) \rightarrow\left(A^{\prime}, C^{\prime}\right)$ be a crucial actions signature morphism and $\mathcal{M}^{\prime}$ be an $\left(A^{\prime}, C^{\prime}\right)$-model. Then, $\sim_{\left(\left.\mathcal{M}^{\prime}\right|_{\sigma}\right)}^{C}=\left.\left(\sim_{\mathcal{M}^{\prime}}^{C^{\prime}}\right)\right|_{\sigma}$. 
Proof. Let us suppose $w \sim_{\left(\left.\mathcal{M}^{\prime}\right|_{\sigma}\right)}^{C} w^{\prime}$. Then, there is an $(A, C)$-bisimulation $B \subseteq$ $\left.W^{\prime}\right|_{\sigma} \times\left. W^{\prime}\right|_{\sigma}$ such that $\left(w, w^{\prime}\right) \in B$. Since for any $c \in C,\left(\left.R^{\prime}\right|_{\sigma}\right)_{c}=R_{\sigma(c)}^{\prime}$, and $\sigma[C]=C^{\prime}$, the relation $B$ is also an $\left(A^{\prime}, C^{\prime}\right)$-bisimulation and, hence $w \sim_{\mathcal{M}^{\prime}}^{C^{\prime}} w^{\prime}$. Moreover, $\left(w, w^{\prime}\right) \in\left(\left.W\right|_{\sigma}\right)^{2}$. Hence $w\left(\sim \sim_{\mathcal{M}^{\prime}}^{C^{\prime}} \cap\left(\left.W^{\prime}\right|_{\sigma}\right)^{2}\right) w^{\prime}$, i.e., $\left.w\left(\sim_{\mathcal{M}^{\prime}}^{C^{\prime}}\right)\right|_{\sigma} w^{\prime}$.

Let us suppose $w\left(\sim_{\mathcal{M}^{\prime}}^{C^{\prime}} \cap\left(\left.W^{\prime}\right|_{\sigma}\right)^{2}\right) w^{\prime}$. Let $B^{\prime} \subseteq W^{\prime} \times W^{\prime}$ be an $\left(A^{\prime}, C^{\prime}\right)$ bisimulation containing $w$ and $w^{\prime}$ (its existence is assured by $w \sim_{\mathcal{M}^{\prime}}^{C^{\prime}} w^{\prime}$ ). Again, since for any $c \in C,\left(\left.R^{\prime}\right|_{\sigma}\right)_{c}=R_{\sigma(c)}^{\prime}$, and $\sigma[C]=C^{\prime}$, we have that $B^{\prime}$ satisfies the conditions of $\left(A^{\prime}, C^{\prime}\right)$-bisimulation. We have also that $\left.W^{\prime}\right|_{\sigma}$ is closed by $A$ actions. Hence, $B^{\prime} \cap\left(\left.W^{\prime}\right|_{\sigma}\right)^{2}$ is an $(A, C)$-bisimulation. Therefore $w \sim_{\left(\left.\mathcal{M}^{\prime}\right|_{\sigma}\right)}^{C} w^{\prime}$.

As a direct consequence of this lemma we have the following result:

Corollary 2. Let $\sigma: A \rightarrow A^{\prime}$ be a function. If $\sigma:(A, C) \rightarrow\left(A^{\prime}, C^{\prime}\right)$ is a crucial actions signature morphism, then $\sigma:\left(A, \sim^{C}\right) \rightarrow\left(A^{\prime}, \sim^{\prime}\right)$ is a behavioural signature morphism.

As a consequence of the last corollary and Lemma 3 we get the functor $\operatorname{Mod}^{C r}:\left(\operatorname{Sign}^{C r}\right)^{o p} \rightarrow \mathbb{C}$ at. Next, by taking $\models_{(A, C)}$ as the satisfaction relation $\models_{(A, \sim C)}$ and instantiating Corollary 1 we have:

Corollary 3 (Satifaction condition for logic of crucial actions). Let $\sigma$ : $(A, C) \rightarrow\left(A^{\prime}, C^{\prime}\right)$ be a crucial actions signature morphism and $\mathcal{M}^{\prime}$ be an $\left(A^{\prime}, C^{\prime}\right)$ model. Then, for any $A$-sentence $\varphi$, we have

$$
\operatorname{Mod}^{C r}(\sigma)\left(\mathcal{M}^{\prime}\right) \models_{(A, C)} \varphi \text { iff } \mathcal{M}^{\prime} \models_{\left(A^{\prime}, C^{\prime}\right)} \operatorname{Sen}^{C r}(\sigma)(\varphi)
$$

Theorem 9 (Crucial actions institution). The tuple $C r=\left(\operatorname{Sign}^{C r}, \operatorname{Sen}^{C r}, \operatorname{Mod}^{C r},\left(\models_{(A, C)}\right)_{(A, C) \in|\operatorname{Sign} C r|}\right)$ is an institution.

Remark 2. As a consequence of Lemma 6 the satisfaction relation $\models_{(A, C)}$ coincides with the observational satisfaction relation $\models_{\sim}$ (see Sect. 2.2) if we use it just for $(A, C)$-models.

Example 3. Coming back to the example considered in Section 1 we want to emphasise that the problem considered there does not apply anymore, if we consider the crucial actions signature morphism $\sigma:(\{a\},\{a\}) \rightarrow(\{a, b\},\{a\})$ with $\sigma(a)=a$. Then, the structure $\mathcal{M}^{\prime}$ in Fig. 2 is not an $(\{a, b\},\{a\})$-model, as explained above, and therefore the reduct w.r.t. $\sigma$ is not meaningful in the crucial actions institution. The situation is different, however, if we consider the model $\mathcal{M}^{\prime \prime}$ of Fig. 3 whose reduct w.r.t. $\sigma$ is just the model $\mathcal{M}$ of Fig. 2 . In this case we have $\mathcal{M}^{\prime \prime} \mid=\sim \downarrow x .\langle a\rangle x$ and $\mathcal{M}=\sim \downarrow x$. $\langle a\rangle x$; see Remark 2 .

According to the results of Sect. 4 we get for free the black-box functor mapping $(A, C)$-models to $A$-models by constructing quotients w.r.t. the $(A, C)$ equalities $\sim_{\mathcal{M}}^{C}$. In particular, we can instantiate Theorem 8:

Corollary 4. For any $(A, C)$-model $\mathcal{M}$ and for any A-formula $\varphi$,

$$
\mathcal{M} \models{ }_{(A, C)} \varphi \text { iff } \mathcal{M} / \sim_{\mathcal{M}}^{C} \models \varphi .
$$




\section{Conclusion and Future Work}

The observational logic with binders $\mathcal{D}^{\downarrow}$ was suggested in [13] as a suitable formalism to develop reactive systems. This research was pursued in [12] with the introduction of an alternative semantics for $\mathcal{D}^{\downarrow}$, endowing it with modal invariance. However, with this accommodation, the satisfaction condition was lost, i.e. unlike the original $\mathcal{D}^{\downarrow}$, this new logic is not an institution. The present paper works on this handicap. As done in the context of the observational semantics (see [15]) we adopted behavioural structures - families of equivalence relations on the states of each model - as behavioural interpretations of the equalities on the states. Then, by adjusting the morphisms of the category of signatures (as done in $[7,3,15])$ the (standard) reduct works properly to assure the satisfaction condition. Under this abstract setting, the black-box functor was defined and the relation between the strict satisfaction of $\mathcal{D}^{\downarrow}$ and the observational ones of $\mathcal{D}_{\sim}^{\downarrow}$ was established. Finally, an interesting instantiation of this generic institution was presented - the Crucial Actions Institution.

, These efforts on the parametrization of the logic with generic observational structures (i), as well on the adjustment of $\mathcal{D}_{\sim}^{\downarrow}$ to recover the institu,tional nature of $\mathcal{D}^{\downarrow}$ (ii) would be worthy explored in the future. Concerning the direction (i), we are looking for a specific observational structur,e (maybe combined with some slight adaptations of $\mathcal{D}_{\sim}^{\downarrow}$ ) to deal with (internal) $\tau$-transitions (e.g. $[14,9]$ ). Moreover, in analogy with what was done in [3] we intend to define an institutional encoding, in sense of [17], from $\mathcal{D}_{\sim}^{\downarrow}$ to $\mathcal{D}^{\downarrow}$. This could provide useful tool support for $\mathcal{D}_{\sim}^{\downarrow}$ borrowed from $\mathcal{D}^{\downarrow}$ - a calculus for $\mathcal{D}^{\downarrow}$ was already suggested in a journal extended version of [13] (currently in revision process). On the direction (ii), it would be interesting to explore the 'once and for all' techniques and results established for generic institutions. In this view, the use and characterisation of the Casl-in-the-large specification constructors [1] in $\mathcal{D}_{\sim}^{\downarrow}$ specifications, as well as the integration of these institutions in HETS [16], could provide appropriate

conditions to make $\mathcal{D}_{\sim}^{\downarrow}$ (and $\mathcal{D}^{\downarrow}$ ) an effective formal method for reactive systems development.

Acknowledgement. We would like to thank the anonymous reviewers of this paper for their careful reviews with many useful comments and suggestions.

\section{References}

1. E. Astesiano, M. Bidoit, H. Kirchner, B. Krieg-Brückner, P. D. Mosses, D. Sannella, and A. Tarlecki. CASL: the common algebraic specification language. Theor. Comput. Sci., 286(2):153-196, 2002.

2. R. Barbuti, N. D. Francesco, A. Santone, and G. Vaglini. Selective mu-calculus and formula-based equivalence of transition systems. Journal of Computer and System Sciences, 59(3):537 - 556, 1999.

3. M. Bidoit and R. Hennicker. Constructor-based observational logic. J. Log. Algebr. Program., 67(1-2):3-51, 2006.

4. T. Braüner. Hybrid Logic and its Proof-Theory. App. Logic Series. Springer, 2010.

5. J. Goguen. Types as theories. In A. W. R. George Michael Reed and R. F. Wachter, editors, Topology and Category Theory in Computer Science. 1991. 
6. J. A. Goguen and R. M. Burstall. Institutions: Abstract model theory for specification and programming. J. ACM, 39(1):95-146, 1992.

7. J. A. Goguen and G. Malcolm. A hidden agenda. Theor. Comput. Sci., 245(1):55$101,2000$.

8. J. A. Goguen and G. Rosu. Hiding more of hidden algebra. In FM'99 - Formal Methods, World Congress on Formal Methods in the Development of Computing Systems, volume 1709 of Lecture Notes in Computer Science, pages 1704-1719. Springer, 1999.

9. J. F. Groote and M. R. Mousavi. Modeling and Analysis of Communicating Systems. MIT Press, 2014.

10. D. Harel, D. Kozen, and J. Tiuryn. Dynamic Logic. MIT Press, 2000.

11. R. Hennicker and M. Bidoit. Observational logic. In A. M. Haeberer, editor, AMAST, volume 1548 of Lecture Notes in Computer Science, pages 263-277. Springer, 1998.

12. R. Hennicker and A. Madeira. Behavioural semantics for the dynamic logic with binders. In M. Roggenbach, editor, Recent Trends in Algebraic Development Methods - Selected Papers of WADT 2016. Springer, (to appear).

13. A. Madeira, L. S. Barbosa, R. Hennicker, and M. A. Martins. Dynamic logic with binders and its application to the development of reactive systems. In A. Sampaio and F. Wang, editors, Theoretical Aspects of Computing - ICTAC 2016, volume 9965 of Lecture Notes in Computer Science, pages 422-440, 2016.

14. R. Milner. Communication and concurrency. PHI Series in computer science. Prentice Hall, 1989.

15. M. Misiak. Behavioural semantics of algebraic specifications in arbitrary logical systems. In Recent Trends in Algebraic Development Techniques, 17th International Workshop, WADT 2004, Revised Selected Papers, volume 3423 of Lecture Notes in Computer Science, pages 144-161. Springer, 2004.

16. T. Mossakowski, C. Maeder, and K. Lüttich. The heterogeneous tool set, Hets. In O. Grumberg and M. Huth, editors, Tools and Algorithms for the Construction and Analysis of Systems (TACAS 2007), volume 4424 of Lecture Notes in Computer Science, pages 519-522. Springer, 2007.

17. A. Tarlecki. Towards heterogeneous specifications. In Frontiers of Combining Systems (FroCoS'98), Applied Logic Series, pages 337-360. Kluwer Academic Publishers, 1998. 